\section{PTEN est un gardien de la sûreté nucléaire}

Julien Thérier, Florence Solari, Marc Billaud
Laboratoire de Génétique moléculaire,

Signalisation et Cancer, UMR 5201,

CNRS, Université Lyon 1, Domaine Rockefeller,

8 , avenue Rockefeller,

69373 Lyon Cedex 08, France.

billaud@univ-lyon1.fr

(K289ع) [4]. De manière intéressante, cette mutation est située dans une boucle comprise entre le domaine catalytique
> PTEN (Phosphatase and TENsin homo$\log$ ) est un gène suppresseur de tumeur très fréquemment muté dans des tumeurs malignes dont l'incidence est élevée dans la population générale [1]. Des mutations germinales de ce gène sont aussi responsables de syndromes héréditaires, en particulier de la maladie de Cowden ${ }^{1}$ caractérisée par une forte prédisposition aux cancers [1]. La protéine PTEN est une phosphatase qui hydrolyse le phosphate situé en position 3 du phosphatidylinositol $(3,4,5)$-triphosphate (PIP3), ce qui lui confère un rôle de régulateur négatif de la voie de signalisation intracellulaire contrôlée par la phosphatidylinositol-3 kinase (PI3K) [2] (Figure 1). Cette activité lipide phosphatase est essentielle à la fonction oncosuppressive de PTEN [2]. Compte tenu de la belle ordonnance de ces résultats et des 2600 articles parus sur ce sujet, que restait-il de vraiment nouveau à apprendre sur PTEN? Deux équipes du Sloan-Kettering à New-York changent notre mode de perception de la fonction de ce suppresseur de tumeur et, dans trois articles parus dans le même numéro de la revue Cell, nous rappellent que les données bien établies ne profitent jamais autant à la science que si le chercheur sait aussi les oublier [3-5].

Un des points de départ de ce travail a été l'analyse des conséquences fonctionnel-

${ }^{1}$ Le syndrome de Cowden, de transmission autosomique dominante, est caractérisé par des hamartomes multiples se formant sur la peau, la poitrine, la thyroïde, le tractus gastro-intestinal, l'endomètre et le cerveau, et par un risque accru de développer des tumeurs malignes (cancers du sein, de l'endomètre ou de la thyroïde). La peau est atteinte dans 90 à $100 \%$ des cas (trichilemmome, papillomatose de la muqueuse orale, kératose acrale et kératose palmoplantaire) [source: Orphanet, orpha 201]. les d'une mutation ponctuelle identifiée dans la maladie de Cowden, qui remplace la lysine 289 par un acide glutamique

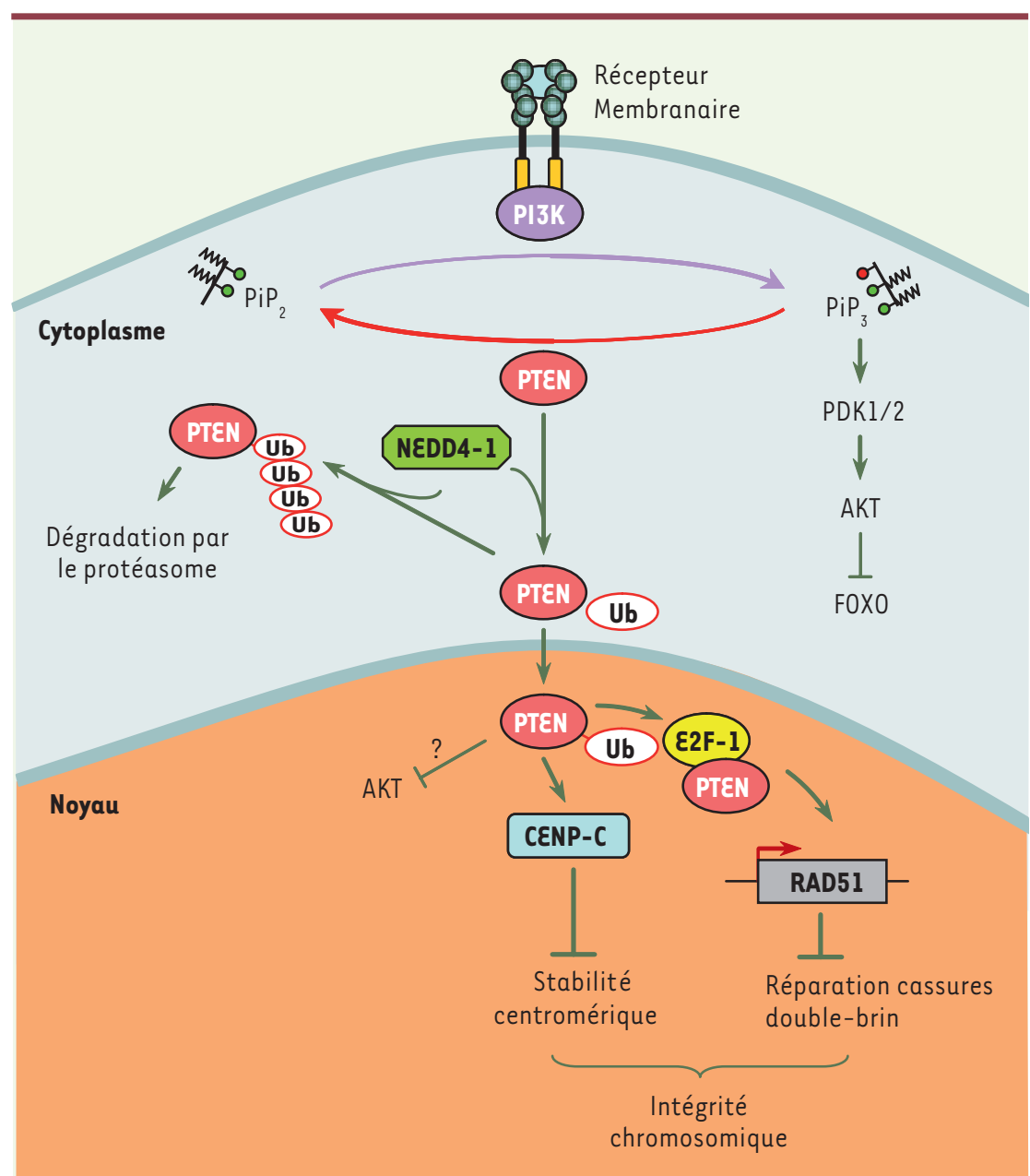

Figure 1. PTEN : un acteur cytoplasmique et nucléaire. Au niveau cytoplasmique, PTEN est connue pour contrôler négativement la voie PI3K/AKT via son action sur le PIP3. La poly-ubiquitinylation de PTEN par la $\varepsilon 3$ ligase NEDD4- 1 contrôle sa dégradation par le protéasome. PTEN mono-ubiquitinylée est adressée au niveau du compartiment nucléaire où elle contrôle l'intégrité chromosomique par deux mécanismes: son association avec la protéine centromérique CENP-C et l'induction de l'expression de la protéine RAD51, en partenariat avec $22 F-1$, impliquée dans les réparations des cassures double-brin. 
et le domaine C2 qui permet le recrutement de PTEN à la membrane plasmique. II s'avère que cette mutation ponctuelle n'altère pas l'activité phosphatase de PTEN, en revanche, la protéine mutante est localisée essentiellement dans le cytoplasme des cellules alors qu'elle est normalement aussi présente dans le noyau. Cette dernière observation a été le fil conducteur des auteurs. On savait déjà que la localisation nucléaire de PTEN était perdue dans les cellules tumorales, et dans certains cas il a même pu être précisément établi que l'intensité du marquage nucléaire de PTEN décroît de l'adénome au carcinome et qu'elle corrèle avec l'index mitotique des cellules cancéreuses [6]. Dans la mesure où l'acide aminé substitué est une lysine, c'est-à-dire un site potentiel d'ubiquitinylation, une des possibilités était que ce type de modification post-traductionnelle puisse contôler la navette nucléo-cytoplasmique de PTEN. De fait, PTEN s'avère ubiquitinylée sur au moins deux sites majeurs, les lysines 13 et 289 [4]. La substitution d'une de ces lysines par un acide aminé de même charge, comme une arginine, inhibe le transport nucléaire de PTEN. Comment l'ubiquitinylation peut-elle contrôler le transport de PTEN ? Si la poly-ubiquitinylation des protéines est plutôt liée à leur dégradation par le protéasome [7], leur mono-ubiquitinylation participe à d'autres processus biologiques comme l'endocytose [8] et les mécanismes de réparation de l'ADN. Effectivement, PTEN est mono-ubiquitinylée dans le noyau et la protéine est stable alors qu'elle est monoubiquitinylée et poly-ubyquitinylée dans le cytoplasme où elle subit une protéolyse par le protéasome, d'où une demi-vie respective de $15 \mathrm{~h}$ et $4,5 \mathrm{~h}$ [4] (Figure 1). L'ubiquitinylation fait intervenir 3 types d'enzymes qui agissent en cascade et l'ubiquitine ligase est celle qui est directement associée à la reconnaissance de sa cible protéique [7]. Les deux équipes ont identifié la protéine NEDD4-1 comme une ubiquitine ligase de la famille HECT (homologue du domaine carboxy-terminal ह6-AP) capable de s'associer spécifiquement à PTEN [3] (Figure 1). La surexpression de NEDD4-1 entraîne une dégradation de PTEN alors que l'inhibition de l'expression de NEDD4-1 avec des ARN interférents stabilise PTEN mais bloque aussi son transport nucléaire [4]. Ces données expérimentales suggèrent que NEDD4-1 pourrait exercer une activité oncogénique en favorisant la dégradation de PTEN. En accord avec cette hypothèse, la surexpression de NEDD4-1 potentialise l'effet de l'oncogène Ras dans des fibroblastes embryonnaires murins invalidés pour le gène suppresseur de tumeur $p 53$ mais n'ont pas cet effet dans ce même type de cellules dans lesquelles les gènes $p 53$ et PTEN ont été inactivés [3]. De plus, dans les tumeurs malignes, il existe une corrélation inverse entre le niveau d'expression de PTEN et celui de NEDD4-1 [3]. Si l'ensemble de ces résultats forme un tout cohérent, la question qui en découle concerne la fonction nucléaire de PTEN. La PI3K, le PIP3 et les kinases effectrices PDKI et AKT sont aussi localisées dans le noyau [6] et les données publiées dans l'un des trois articles de Cell suggèrent qu'une forme de PTEN uniquement nucléaire est capable de bloquer l'activation d'AKT [4]. Mais le mécanisme moléculaire n'est pas décrit et pourrait ne pas nécessiter l'activité lipide phosphatase de PTEN puisque le PIP3 nucléaire n'est pas sensible à l'action de cette phosphatase [9]. C'est à ce stade que s'ouvre le troisième volet du triptyque. En exploitant des fibroblastes dans lesquels les deux allèles de PTEN sont invalidés, les auteurs montrent que ces cellules sont le siège d'une instabilité chromosomique importante [5]. Ces fibroblastes présentent des aberrations chromosomiques spontanées et en particulier des anomalies centromériques. En accord avec ces données, dans les cellules normales, PTEN se révèle être localisée aux centromères et s'associe spécifiquement avec la protéine CENP-C du kinétochore [5] (Figure 1). Fait encore plus remarquable, l'expression d'une forme de PTEN tronquée dans sa région carboxyterminale, qui n'interagit plus avec CENP$C$ mais conserve son activité phosphatase, agit comme un dominant-négatif sur PTEN sauvage et induit une fragmentation des centromères. Les résultats obtenus par deux équipes indiquent également que PTEN participe à la réparation des cassures double-brin de I'ADN en régulant l'expression du gène codant le facteur Rad51, un élément crucial d'un complexe multi-protéique participant au processus de recombinaison homologue lors de la réparation des dommages de I'ADN. PTEN s'associe au facteur de transcription $\varepsilon 2 F 1$ et régule l'expression de Rad5l via sa fixation indirecte sur la région promotrice de ce gène [5] (Figure 1).

PTEN est donc une protéine dont le transport nucléo-cytoplasmique est contrôlé par ubiquitinylation et dont les fonctions cytoplasmiques et nucléaires apparaissent différentes. Dans ce travail, le maintien de la stabilité génomique ne dépend pas de l'activité phosphatase de PTEN, quoique des mécanismes associés à la préservation de la stabilité du génome et requérrant l'activité enzymatique de PTEN aient aussi été décrits [10]. Les modes de régulation de PTEN ne sont pas sans rappeler ceux de la protéine onco-suppressive $\mathrm{p} 53$, et si l'on se réfère à cette dernière, nul doute que d'autres effecteurs régulant I'ubiquitinylation et d'autres types de modifications post-traductionnelles modulant la fonction biologique de PTEN restent encore à caractériser et feront l'objet de nouvelles synthèses. $\diamond$ PTEN: a guard of nuclear safety

\section{RéFÉRENCES}

1. Eng C. PTEN, one gene, many syndromes. Hum Mutat 2003; 22 : 183-98.

2. Sulis ML, Parsons R. PTEN: from pathology to biology. Trends Cell Biol $2003 ; 13$ : 478-83.

3. Trotman LC, Wang X, Alimonti A, et al. Ubiquitination regulates PTEN nuclear import and tumor suppression. Cell $2007 ; 128$ : 141-56.

4. Wang X, Trotman LC, Koppie T, et al. NEDD4- 1 is a proto-oncogenic ubiquitin ligase for PTEN. Cell 2007 ; $128: 129-39$.

5. Shen WH, Balajee AS, Wang J, et al. Essential role for nuclear PTEN in maintaining chromosomal integrity. Cell 2007 ; $128: 157-70$.

6. Lian Z, Di Cristofano A. Class reunion: PTEN joins the nuclear crew. Oncogene $2005 ; 24$ : 7394-400.

7. Coux 0 , Piechaczyk M. Le système ubiquitine/ protéasome : un ensemble de complexes pour dégrader les protéines. Med Sci (Paris) 2000 ; 16 : 623-9.

8. Brou C, Logeat F. Endocytosis and Notch signalling Med Sci (Paris) 2006 ; 22 : 685-8.

9. Lindsay $Y$, McCoull D, Davidson L, et al. Localization of agonist-sensitive Ptdlns $(3,4,5)$ P3 reveals a nuclear pool that is insensitive to PTEN expression. J Cell Sci 2006 ; $119: 5160-8$

10. Puc J, Keniry M, Li HS, et al. Lack of PTEN sequesters CHKl and initiates genetic instability. Cancer Cell $2005 ; 7$ : 193-204. 